\title{
„Nicht voreilig entscheiden“
}

Interview mit Jan-Philipp Schmidt, Vorsitzender des Bundesverbands der zahnmedizinischen Alumni in Deutschland (BdzA)

djz: Welche Trends beobachten Sie in der Weiterbildung und den Spezialisierungen bei den jungen Zahnärzten zurzeit?

- Schmidt: Die Kolleginnen und Kollegen wägen inzwischen sehr genau $\mathrm{ab}$, ob eine Spezialisierung im Kosten-Nutzen-Verhältnis für sie Sinn macht. Yor zwei bis drei Jahren war unseres Erachtens der "run" auf die damals angebotenen Master-Kurse und Curricula unüberlegter.

djz: Es gibt langsam immer mehr Master-Studiengänge. Wie können sich Interessenten orientieren?

- Grundsätzlich begrüßt der BdZA sämtliche Fort- und Weiterbildungen, die sich an die Rahmenbedingungen der Bundeszahnärztekammer (BZÄK), der Deutschen Gesellschaft für Zahn-, Mund-und Kieferheillkunde (DGZMK) und der Vereinigung der Hochschullehrer fur Zahn-, Mund- und Kiefer- heilkunde (VHZMK) halten. Der BdZA wird ein Online-Kompendium der Master-Kurse erstellen, das zur Internationalen Dental-Schau (IDS) 2011 unter www.dents, de allen interessierten Zahnmedizinern zu Verfügung steht.

djz: Raten Sie jungen Zahnärzten, sich früh zu spezialisieren?

- Ich persönlich habe noch innerhalb meiner Assistenzzeit einen Master-Kurs absolviert und kann jungen Kolleginnen und Kollegen nur raten, die Entscheidung zur Spezialisierung gut zu überdenken und nicht voreilig zu treffen. Die Kosten für Master-Kurse und Curricula sind bei den dürftigen Assistenzgehältern doch sehr hoch. Sinnvoll ist sicherlich, mindestens ein Jahr allgemeinzahnärztlich tätig zu sein, um einen guten Überblick der Fachgebiete im Praxisalltag kennenzulernen. Wer feststellt, dass alle Bereiche interessant sind, soll doch gern Generalist werden und sich regelmäßig im Rahmen yon Messen sowie Fachkongressen auf dem aktuellen Stand der Wissenschaft halten - ein Zwang zu Master \& Co, ist gottlob in Deutschland nicht absehbar und auch nicht der einzige Weg zu Glück und Erfolg.

djz: Sind bei der Fortbildung einzelne Anbieter vorzuziehen, etwa die

Kammern oder die APW oder privatwirtschaftliche Dentalschulen?

- Ich denke, dass man hier kein pauschales Urteil fällen kann. Wichtig ist, dass die Veranstaltungen modular aufgebaut sein sollen und sich im Sinne des Bologna-Prozesses am ECTS (European Credit Transfer and Accumulation System) orientieren. Damit ist es zum Beispiel möglich, sich Leistungen aus Curricula in Form von ECTS-Punkten aufMaster-Kurse etc, anrechnen zulassen. Die so gewonnene Flexibilität ist ein großer Pluspunkt für alle jungen Kolleginnen und Kollegen. 